the historical appellation apudomas for tumours of the neuroendocrine system. they appear to be unaware that the accepted terminology for these is now neuroendocrinoma and neuroendocrine carcinoma.

Part 2 of the work has 19 chapters, each embracing a single cell type or a family of related cells. There are thus too many cells and too many chapters for pertinent comment here. Coverage of each cell type sensibly follows a more or less uniform pattern: distribution, products, innervation, electrophysiology, ontogeny and phylogeny. In Chapter 13, curiously, the thyroid parafollicular cells are referred to as "C (from clear) cells", instead of the usual and correct view that "C" stands for Copp's calcitonin! But the oddest inclusion is the subject of Chapter 27, the mast cell. Here its accepted mesenchymal origin is emphasized as typifying the authors' view (p.85) on the derivation of paraneurons from all the germ layers.

Having come this far, there may still be some readers inclined to ask why it was necessary to write this book at all, except as an illustrated text for students of anatomy. Yet perusal of the preface alone provides some insight. Its variety of hopeful expressions, and its oblique references to the works of Starling, Lyell and Darwin, clearly anticipate the forthcoming descent of the mantle of these great men upon the authors of The Paraneuron. Those who read further will have no trouble in detecting the vested interest of this reviewer, whose published contributions to neuroendocrinology are referred to selectively and frequently, though skilful handling of relevant literature is not restricted to the work of any one author or group of authors. With respect to my own contributions, the paper quoted is invariably that bearing the latest possible date of publication. Thus is effectively elided the chronological distance between the APUD concept (the neuroendocrine system) and its paraneuronal equivalent (see Nova acta Leopoldina 56, 118-124, NF255; 1984).

Composed as it is, with the benefit of hindsight, it is necessary to conclude that The Paraneuron is neither more nor less than a gigantic take-over bid for the equity of that large section of neurobiology which has hitherto been called neuroendocrinology. Obviously I am not prepared to recommend the book to those unversed in this field; but for those who are, it makes interesting reading.

The choice between neuroendocrinology and paraneuronics is a clear one, and I imagine that the distinction will be maintained without difficulty.

Anthony G.E. Pearse is Emeritus Professor of Histochemistry in the University of London and Consultant Pathologist to Hammersmith Hospital (the Royal Postgraduate Medical School), Du Cane Road, London WI2 OHS, UK.

\section{Stellar systems in theory}

\section{Frank Shu}

Galactic Dynamics. By James Binney and Scott Tremaine. Princeton University Press: 1988. Pp. 733. Hbk \$75, £42.10; pbk $\$ 25, £ 14$.

SINCE 1970 or so, study of the structure, dynamics and evolution of stellar systems such as galaxies and star clusters has undergone a renaissance. Books on the subject have become badly outdated, and a new treatment has long been overdue. The new graduate text by James Binney and Scott Tremaine, two of the world's leading practitioners in this field, fills the gap admirably. The manuscript circulated for a few years while undergoing preparation and revision, and among the cognoscenti it has already achieved the status of a classic. Now it is available as an attractive volume from Princeton University Press; the resultant product has been well worth the wait.

The book begins with an observational overview, and then dives deeply, with a minimum of splash, into authoritative discussions of virtually the entire range of topics currently being worked on: potential theory (mass models, that is, densitypotential pairs), orbits and integrals of motion, dynamics of collisionless systems (equilibria and stability), spiral structure, stellar encounters, dynamical friction and mergers, theory of star clusters (evaporation, core collapse and the effects of binaries), galactic evolution (chemical as well as in colour and luminosity) and the role of dark matter in the Universe. Except for the last two chapters, the emphasis throughout is heavily theoretical, with relatively little cross-referencing to the companion observational volume Galactic Astronomy written by Mihalas and Binney (published by W. H. Freeman). Nevertheless, the discussions are well motivated from the physical and astronomical points of view, and they are self-contained, the more technical manipulations being banished to a series of mathematical appendices. For material too complex to present in a textbook format, readers are referred to the original papers in the research literature, after having been given an introduction at a level that has adequately prepared them to profit from such reading.

The strengths of the book are many. In discussing the work of others, the authors have not been content merely to reproduce the original arguments, but have reworked them to make the exposition more transparent or closer to the developments in the rest of the book. For example, their derivation of Toomre's sequence of mass models for galactic disks, starting with a simple physical visualization of Kuzmin's disk, is a model of elegance and brevity. Binney and Tremaine also take potentially complex topics - such as the phase-space structure of regular and irregular orbits, the equilibria of triaxial galaxies, the cannibalism of a small galaxy by a larger host, or the evolution of globular clusters - and reduce them to their dynamical essentials without losing the flavour of the richness of the physical phenomenon. Their handling of more incompletely understood problems, such as the nature of and evidence for dark matter, is equally concise, while remaining well balanced, up to date and full of insight.

The book does have its eccentricities. For example, the method of characteristics is never introduced per se as a standard technique for solving quasilinear partial differential equations, of which the 'collisionless' Boltzmann equation is a prime example. Thus, the authors' demonstration of the mathematical relationship between the constants of motion derived from orbit analyses and the most general solution for the distribution function of an encounterless stellar system shows sufficiency, but not necessity, and deprives the reader of a useful tool of the trade. Also, although Binney and Tremaine generally do an excellent job of presenting all sides of controversial topics, their judgement is occasionally coloured in favour of work that has been carried out at Princeton and the two Cambridges (places where they have spent appreciable time). For example, the acerbic comment on p. 607 that "The mass-to-light ratio of binary galaxies is probably large, but not so large as the ratio of the masses of papers on this subject to the light they have shed on it" does a disservice to a serious subject. Fortunately, such slips of taste are rare, and the authors usually make intelligent and positive use of the opportunity, expressed in their preface, "to advertise [their] own views on the proper organization and relative importance of various topics and areas".

For a comprehensive and penetrating treatment of modern problems of the structure and evolution of stellar systems, this book has no peers. I strongly suspect that copies of Galactic Dynamics will find permanent homes not only in libraries, but in the offices and studies of all serious students of the field.

Frank Shu is a Professor in the Department of Astronomy, University of California, Berkeley, California 94720, USA

- The latest volume in Cambridge University Press's astrophysics series is $X$-ray Emissions from Clusters of Galaxies, by Craig L. Sarazin. The book deals largely with the observational properties of X-ray clusters of galaxies and with the theory of the physical state, dynamics and origin of the hot intracluster gas. Price is $£ 32.50, \$ 49.50$. 